\title{
Human lumbosacral root and ligamentum flavum thicknesses: a magnetic resonance study
}

\author{
R. Khasawneh¹ㅁ, E. Abu El-Rub¹, M. Allouh²,3 \\ ${ }^{1}$ Department of Basic Medical Sciences, Faculty of Medicine, Yarmouk University, Irbid, Jordan \\ 2Department of Anatomy, Faculty of Medicine, Jordan University of Science and Technology, Irbid, Jordan \\ ${ }^{3}$ Department of Anatomy, College of Medicine and Health Sciences, United Arab Emirates University, Al Ain, \\ United Arab Emirates
}

[Received: 3 July 2021; Accepted: 18 October 2021; Early publication date: 9 November 2021]

\begin{abstract}
Background: This study investigated the lumbosacral plexus (LSP) nerve root thickness and ligamentum flavum (LF) thickness and correlated them with age and sex. These findings provided a useful data for spinal nerve root micro-anastomosis surgery and lumbar decompression surgery.

Materials and methods: This retrospective study was conducted with 350 individuals with ages ranging from 21 to 80 years under magnetic resonance imaging to evaluate the possible cause of a lower back pain.

Results: According to the morphometric measurements of the LSP root thickness, the diameter gradually increased from $L 1$ to S1. L1 has the thinnest root (3.9 \pm $\pm 0.81 \mathrm{~mm}$ ) while $\mathrm{S} 1$ has the thickest root $(5.45 \pm 0.8 \mathrm{~mm})$. The measurements revealed inconsiderable differences in the LSP thickness in relation to age, sex in the study population. Regarding the $L F$, the thickness of the $L F$ was found to insignificantly increase with age. Besides, the LF thickness was inconsequentially higher in female. The mean thickness of the right $L F$ at different spinal levels was measured $(L 2-L 3=3.19 \pm 0.27, L 3-L 4=3.38 \pm 0.11 \mathrm{~mm}, L 4-L 5=3.71 \pm$ $\pm 0.29 \mathrm{~mm}$, and $L 5-S 1=3.64 \pm 0.21 \mathrm{~mm})$. The mean thickness of the left $L F$ was non-significantly higher.

Conclusions: The LSP root and LF thicknesses not related to age or sex. (Folia Morphol 2023; 82, 1: 72-78)
\end{abstract}

Key words: lumbosacral plexus, ligamentum flavum, spinal nerve root, magnetic resonance image

\section{INTRODUCTION}

The lumbosacral plexus (LSP) is a series of nerve convergences and divergences that ultimately combine into larger terminal nerves and supply the pelvis and lower extremities

Disorders affecting the LSP with time may alter root diameter such as lumbosacral radiculopathy which is a damage that caused by compression of the nerve roots which exit the spine, levels L1-S4 and cause a pain in the lower back and hip which radiates down the back of the thigh into the leg [3]. Determine normal LSP nerve root dimensions can provide valuable structural information that for monitoring any pathological changes of the lumbar nerve root [20]. Moreover, nerve root size may also determine degree of blockade after epidural

Address for correspondence: Assist. Prof. R. Khasawneh, Department of Basic Medical Sciences, Faculty of Medicine, Yarmouk University, Irbid, Jordan, e-mail: ramada@yu.edu.jo

This article is available in open access under Creative Common Attribution-Non-Commercial-No Derivatives 4.0 International (CC BY-NC-ND 4.0) license, allowing to download articles and share them with others as long as they credit the authors and the publisher, but without permission to change them in any way or use them commercially. 
or spinal anaesthesia, but good measures of this fundamental anatomic parameter have not been published.

Numerous factors can affect the LSP thickness; these factors can be focal like tumoural factors or can be diffused like diabetes-related factors. Pathological changes pertaining to LSP may alter nerve root thickness [14]. Slightly increased thickness is sometimes difficult to assess specially when changes are diffused and symmetrical in both sides. Furthermore, there are some controversies in defining the normal LSP thickness. To date, few studies addressed the normal LSP root size dimensions and how they are affected by different diseases [8].

In the light of this, the first purpose of the current study is to establish normal LSP nerve root thickness retrospectively by reviewing large series of magnetic resonance imaging (MRI) images, and correlate the LSP thickness with age and gender. Knowing the thickness of the LSP has clinical significance for the surgeons who will undergo surgical intervention for LSP or the surrounding structures. The available literature regarding LSP dimensions has some contradictions regarding the exact thickness of the LSP $[12,20]$.

Also to make this article more value, we investigated the ligamentum flavum (LF). The LF wraps around the medial aspect of the spinal articulations and has high percentage of elastic fibres which is responsible of its yellowish colour. These elastic characteristics of LF diminish with age, due to remarkable loss of the elastic fibres and a concomitant replacement by collagen fibres [17]. Moreover, LF joints between vertebrae, LF reinforced and supported the vertebrae as it attached to the front of the upper lamina above and to the back of the lower lamina below [27], also, LF maintain a smooth surface of the posterior dural sac, control intervertebral movement, and affect the intrinsic stability of the spine [19].

Many researchers have demonstrated that the LF plays an important role in vertebral disease, more specifically, in spinal stenosis $[7,28]$. The LF thickening is considered an important cause of radiculopathy exists in lumbar degenerative disease [23]. The LF is an important anatomical structure, and might be changed in patients suffered from low back or leg pain. Therefore, the thickness of the LF should be measured and evaluated carefully in the case of spinal stenosis. Considering this fact, we studied the LF on each side of different spinal levels, and correlated the measurements with age and sex.

\section{MATERIALS AND METHODS}

\section{Sample study}

This retrospective study first was performed with the Ethics approval of the institutional research board at Jordan University of science and technology (IRB \# 8/134/2020).

The study included participants from King Abdullah University hospital in Irbid, Jordan, who had been referred for MRI imaging to assess the possible causes of low back pain in the past two years. Individuals with a history of fractures, dislocation or surgeries in the vertebral column or spinal cord were excluded from the study. The total study group consisted of 350 individuals with ages ranging from 21 to 80 years. Among the study subjects, 155 were males and 195 were females.

The male and female individuals were further subcategorized into two age groups; the young age group (21-50 years) and the elderly age group (51-80 years).

The control group included in the current study composed of 85 healthy individuals who underwent MRI as a routine procedure of their annual checkup. The subjects in the control group were without structural changes in the vertebral column similar to the study group, but had no pain. This control group was included in the current study to exclude any possible differences that may result from inflammatory or degenerative changes. Among the control group, 41 were females and 44 were males, with age range from 25 to 57 years.

\section{MRI imaging measurements}

Images were obtained on a Signa HDxt 3.0T scanner. For the LSP, IDEAL T2 weight images on the coronal plane were acquired for all studied individuals. The T band width: $62.5 \mathrm{kHz}$, matrix was $320 \times 256$, with $42 \times 42 \mathrm{~cm}$ field of view. The slice thickness was $1.5 \mathrm{~mm}$, gap: $0 \mathrm{~mm}$, repetition time: $7680 \mathrm{~ms}$, echo time: $90.8 \mathrm{~ms}$, band width: $62.5 \mathrm{kHz}$, number of excitations: 3.

Observations were made on the DICOM files using the manufacture's software. The LSP root size was evaluated by two specialised radiologists, who were blind to the study. We measured LSP root diameter from the first lumbar (L1) to the first sacral (S1) nerve on the coronal plane at $5 \mathrm{~mm}$ distance from the dorsal root ganglion (Fig. 1).

Regarding LF, T1-sequence axial sections at four levels: L2-L3, L3-L4, L4-L5, and L5-S1 were obtained. 


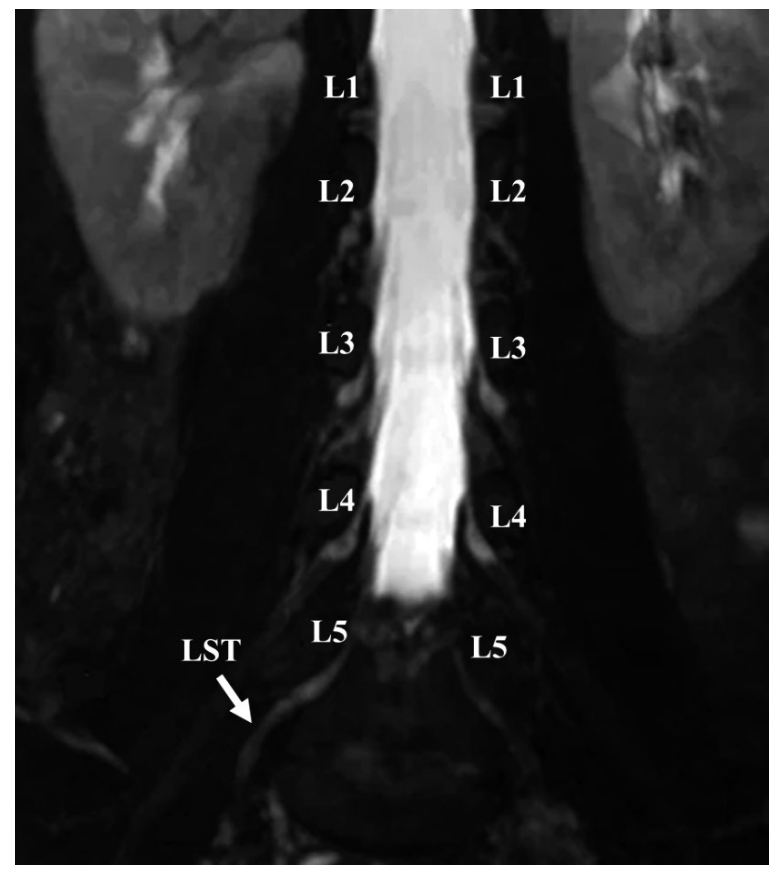

Figure 1. Magnetic resonance image of a coronal section demonstrating the method for determining the lumbosacral plexus roots from L1 to S1; LST — lumbosacral plexus.

The same radiologists who investigated the LSP also assessed the thickening of the LF in all study subjects. The thickness of the LF was measured with an electronic ruler with a resolution of $0.1 \mathrm{~mm}$ by means of a line drawn transversally to the facet joint level (Fig. 2) through the middle section of the LF. If the thickness was bilaterally asymmetrical, the measurement of the thickest part was used.

\section{Statistical analysis}

After applying the Levene's test to determine the homogeneity of variance, the data were evaluated by independent samples t-test or one-way analysis of variance (ANOVA) at $5 \%$ and $1 \%$ levels of significance. Fischer's (LSD) post hoc test was performed to examine statistical differences between the groups. The data were presented as mean \pm standard error of the mean (SEM).

\section{RESULTS}

\section{Lumbosacral root thickness}

The results of MRI images and basic anatomy atlas showed that the conus medullaris terminate at the level of L1-L2 as described by previous study [13].

The results also showed insignificant difference in the thickness of lumbosacral roots between the
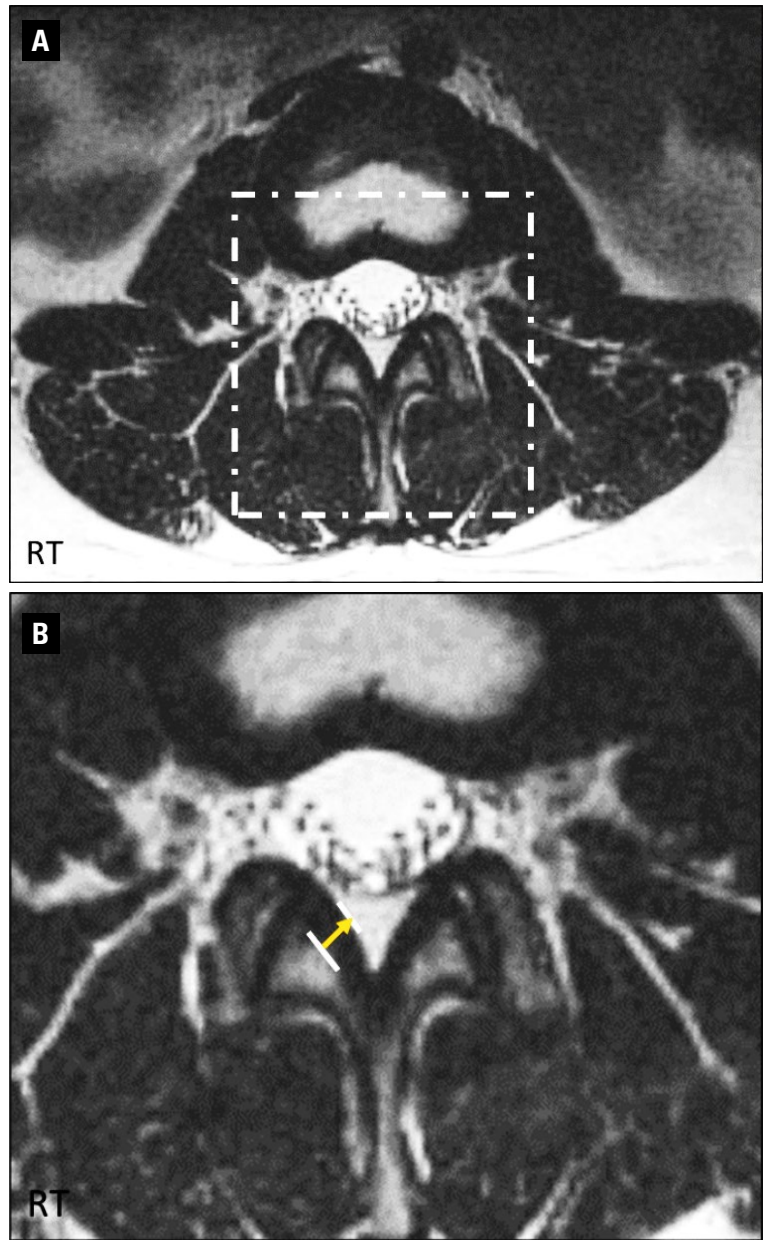

Figure 2. Measurement of the thickness of the ligamentum flavum in T1-weighted magnetic resonance image scan; $\mathbf{A}$. The target area was selected for analysis; $\mathbf{B}$. The arrow represents the ligamentum flavum thickness at the facet joint level; RT — right side.

right and left side and the mean values of the root thickness in our patients were as follows: L1: $3.9 \pm$ $\pm 0.81 \mathrm{~mm}, \mathrm{~L} 2: 4.17 \pm 0.79 \mathrm{~mm}, \mathrm{~L} 3: 4.3 \pm 0.78$, L4: $5.2 \pm 0.72 \mathrm{~mm}$, L5: $5.32 \pm 0.45 \mathrm{~mm}$ and S1: $5.45 \pm 0.8 \mathrm{~mm}$. The diameters of each spinal nerve root are shown in Table 1.

The data were collected from patients without structural changes in vertebral column but having pain. In order to exclude any possible effects that may results from pain and inflammation, additional data were collected from 85 healthy individual without structural changes in the vertebral column and having no pain. The results did not show any significant difference in the thickness of lumbosacral root between the study group and the control group. Our data indicated and validated that chronic inflammatory pain does not affect the structure and morphology of the vertebral column. 
Table 1. The diameters of lumbosacral nerve roots

\begin{tabular}{lccc}
\hline $\begin{array}{l}\text { Lumbosacral } \\
\text { root }\end{array}$ & $\begin{array}{c}\text { Right root } \\
\text { thickness [mm] }\end{array}$ & $\begin{array}{c}\text { Left root } \\
\text { thickness [mm] }\end{array}$ & $\begin{array}{c}\text { Root thickness } \\
\text { [mm] }\end{array}$ \\
\hline L1 & $3.9 \pm 0.94$ & $3.9 \pm 0.68$ & $3.9 \pm 0.81$ \\
L2 & $4.17 \pm 0.76$ & $4.17 \pm 0.82$ & $4.17 \pm 0.79$ \\
L3 & $4.3 \pm 0.95$ & $4.31 \pm 0.79$ & $4.3 \pm 0.87$ \\
L4 & $5.2 \pm 0.57$ & $5.25 \pm 0.88$ & $5.2 \pm 0.72$ \\
L5 & $5.32 \pm 0.54$ & $5.32 \pm 0.38$ & $5.32 \pm 0.45$ \\
S1 & $5.5 \pm 0.78$ & $5.4 \pm 0.83$ & $5.45 \pm 0.8$ \\
\hline
\end{tabular}

Data are shown as mean \pm standard error of the mean.

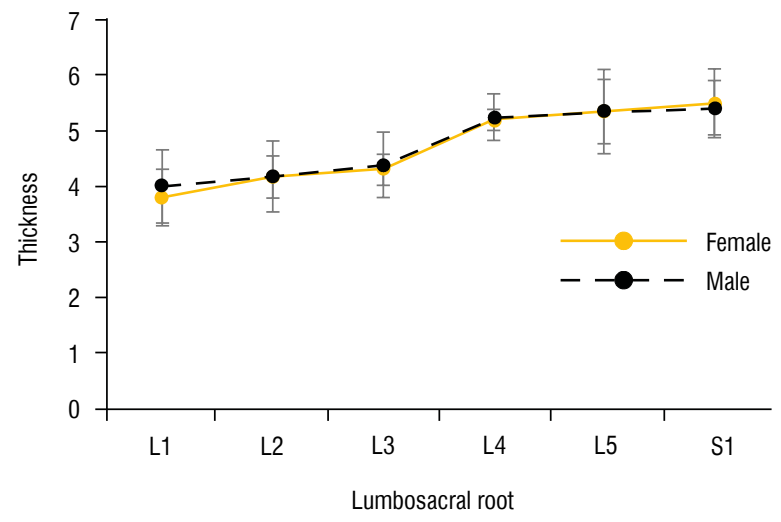

Figure 3. Variation of lumbosacral plexus (LSP) nerve roots thickness with sex. The data revealed a non-significant difference in the LSP nerve roots thickness between males and females. Each column represents the mean LSP nerve root thickness \pm standard error of the mean.

\section{Variation of lumbosacral root thickness with sex and age}

The subjects were divided according to their gender as follows: male group $(n=155)$ and female group $(n=195)$. We found no correlation $(p=0.43)$ between the lumbosacral root thickness and gender of the participants (Fig. 3).

The subjects were further divided into two different age subgroups as follows: young adults (21-50 years; $n=118$ ), and elderly group (51-80 years, $n=232)$. There was no noticeable association ( $p=0.31)$ between the thickness of the lumbosacral roots and age of study subjects (Fig. 4).

\section{Variation of ligamentum flavum with sex and age}

The thickness of LF was measured at L2-3, L3-4, L4-5 and L5-S levels on both sides. The results are showed in Table 2. The relationship among thickness, age, and spinal level was examined.

There was no significant difference between the two sex groups pertaining to LF thickness at the

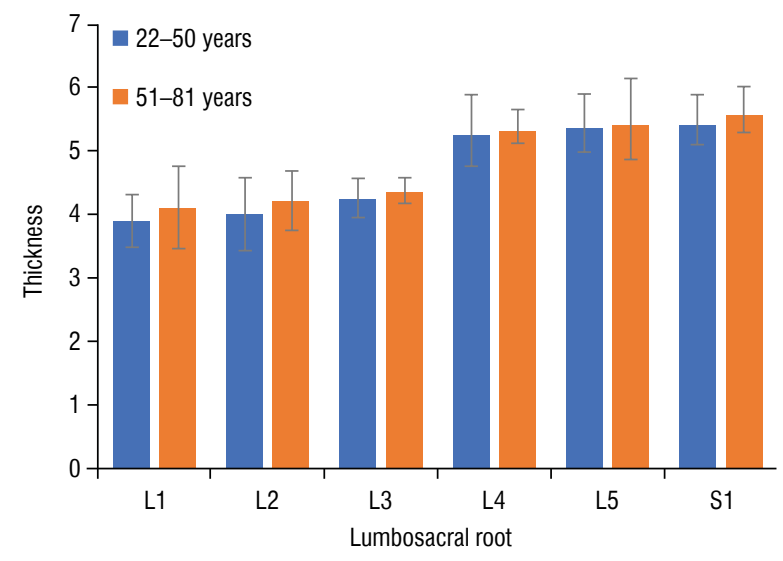

Figure 4. Variation of lumbosacral plexus (LSP) nerve roots thickness with age. The data revealed a non-significant difference in the LSP nerve roots thickness between young and older populations. Each column represents the mean LSP nerve root thickness \pm standard error of the mean.

Table 2. Measurement of thickness of the ligamentum flavum (LF) at different spinal levels

\begin{tabular}{lcc}
\hline LF spinal level & Right side [mm] & Left side [mm] \\
\hline L2-L3 & $3.19 \pm 0.27$ & $3.47 \pm 0.2$ \\
L3-L4 & $3.38 \pm 0.11$ & $3.51 \pm 0.21$ \\
L4-L5 & $3.71 \pm 0.29$ & $3.84 \pm 0.15$ \\
L5-S1 & $3.64 \pm 0.21$ & $3.73 \pm 0.17$ \\
\hline
\end{tabular}

Data are shown as mean \pm standard error of the mean.

segments $L 2 / 3, L 3 / 4, L 4 / 5$ and $L 5 / S 1$ ( $p>0.05$ ). On the other hand, females found to have larger average thickness of the right and left LF, and the greater average thickness of the ligament was at L3/L4 (Table 3).

According to age, our results indicated that LF thickness increased with age; but this increase was insignificant. However, the increments at L4-5 and L3-4 were larger than that at L2-3 and L5-S1. Moreover, the results showed that the mean thickness of the left $L F$ was higher in the left side than the right side (Table 4).

In this context, comparing the study group with the control group did not show any significant difference in the thickness of both right and left LF. The data are shown in Supplementary material (see journal website).

\section{DISCUSSION}

The LSP is a well-protected structure because of its secure location deep in the retroperitoneum, protected by the pelvic brim, which will give the required support and firmness for LSP [6]. Based on that secured and unique structure of LSP, the injuries of 
Table 3. Thickness of ligamentum flavum (LF) at different lumbar spinal levels in different sex groups

\begin{tabular}{lccc}
\hline LF level & Male & Female & P-value \\
\hline Right side & & & \\
L2-L3 & $3.19 \pm 0.22$ & $3.25 \pm 0.24$ & 0.44 \\
L3-L4 & $3.31 \pm 0.17$ & $3.38 \pm 0.12$ & 0.28 \\
L4-L5 & $3.48 \pm 0.27$ & $3.4 \pm 0.19$ & 0.12 \\
L5-S1 & $3.67 \pm 0.2$ & $3.53 \pm 0.34$ & 0.62 \\
Left side & & & \\
L2-L3 & $3.54 \pm 0.11$ & $3.6 \pm 0.28$ & 0.39 \\
L3-L4 & $3.59 \pm 0.29$ & $3.68 \pm 0.17$ & 0.14 \\
L4-L5 & $3.68 \pm 0.14$ & $6.76 \pm 0.23$ & 0.52 \\
L5-S1 & $3.62 \pm 0.23$ & $3.7 \pm 0.25$ & 0.29 \\
\hline
\end{tabular}

Data are shown as mean \pm standard error of the mean.

Table 4. Thickness of ligamentum flavum (LF) at different lumbar spinal levels in different age groups

\begin{tabular}{lccc}
\hline LF level & $\mathbf{R T}$ & $\mathbf{L T}$ & P-value \\
\hline 22-50 years & & & \\
L2-L3 & $3.19 \pm 0.15$ & $3.37 \pm 0.3$ & 0.51 \\
L3-L4 & $3.3 \pm 0.24$ & $3.45 \pm 0.17$ & 0.18 \\
L4-L5 & $3.54 \pm 0.31$ & $3.73 \pm 0.19$ & 0.25 \\
L5-S1 & $3.49 \pm 0.11$ & $3.64 \pm 0.24$ & 0.12 \\
51-80 years & & & \\
L2-L3 & $3.27 \pm 0.27$ & $3.46 \pm 0.16$ & 0.34 \\
L3-L4 & $3.36 \pm 0.23$ & $3.51 \pm 0.32$ & 0.27 \\
L4-L5 & $3.67 \pm 0.15$ & $3.82 \pm 0.14$ & 0.15 \\
L5-S1 & $3.57 \pm 0.09$ & $3.7 \pm 0.32$ & 0.62 \\
\hline
\end{tabular}

Data are shown as mean \pm standard error of the mean. LT — left side; RT — right side

lower extremities are less compared with the upper extremities.

The exact values of human LSP thickness are varied in the literature. The clinical importance of morphometric data on LSP thickness has been emphasized by many studies $[4,11,12,15,20,29]$. In this retrospective study, the thickness of LSP showed a gradual increase from L1 to S1, known as cephalo-caudal pattern (Table 1). Large variations in determining LSP thickness should be taken into consideration when performing morphometric analyses of the spinal cord and the LSP. Based on such morphometric data, the existence of pathological conditions in the LSP such as compression or atrophy can be assessed by comparing these pathological changes with the normal reported values of the affected segment. The present study provides an updated reference of the normal
LSP thickness values and its relation to the most important risk; age and sex. This reference can be used to evaluate any disease affecting the LSP area.

The outcome of this study revealed that the LSP thickness increases by moving downwards, towards the sacrum as previously reported $[4,11,29]$. The only study that contradicts the concept of gradual increase in LSP thickness was conducted by Izci et al. [12] who reported that L1 was the thinnest root of the LSP $(4.1 \mathrm{~mm})$ and L4 was the thickest $(5.5 \mathrm{~mm})$ root of the LSP. No study so far investigated the effect of age or sex on the thickness of LSP, which has been revealed by our study to have no significant relation to the thickness of the LSP.

In this study we were able to measure the diameters of all lumbosacral spinal nerve roots using the 2-point Dixon deconvolution (IDEAL T2-WI) technique to separate water and lipid resonance signals by phase-sensitive MRI. The IDEAL T2-WI is used to obtain an inhomogeneous map based on only in-phase and out-of-phase image data. The method 2-point Dixon technique is designed for T1- or density-weighted spinecho imaging while the double-echo scheme is more appropriate for T2-weighted spin-echo imaging [5]. The 2-point Dixon deconvolution technique is considered superior to other techniques, such as short tau inversion recovery (STIR) sequence, in evaluating the nerve structure and distinguish nerve roots from peripheral tissue [25]. T2 Dixon water-only image showed higher mean scores for fat suppression quality and lesion conspicuity than SPAIR [18], moreover, 2-point Dixon fat suppression was significantly more consistent than SPAIR on both T2 and T1 [10].

The LF is a short but thick ligament of elastic fibres that connects the laminae of adjacent vertebrae from the $C 2$ to $S 1$ [1]. The function of LF to maintain the upright posture and to assist the vertebral column in resuming its shape after flexion, and maintains a smooth surface for the dural sac [26]. The results of this study did not show any correlation between age or sex and the LF thickness in both sides. On the other hand, minor changes in LF thickness were beheld at the L4-L5 and L5-S1 spinal levels as age increased. Moreover, LF was thicker in females comparing to males. The finding of this study was in congruence with other studies [16, 26]. The values of LF reported in the current study were similar to those reported by Horwitz [9], who reported that $\mathrm{LF}$ thickness were $(\mathrm{L} 3-\mathrm{L} 4=3.5 \mathrm{~mm}$, $\mathrm{L} 4-\mathrm{L} 5=3.8 \mathrm{~mm}$, and $\mathrm{L} 5-\mathrm{S} 1=3.6 \mathrm{~mm})$. The highest 
value reported was $6.1 \mathrm{~mm}$ by Ramani et al. [24], who explained their findings by suggesting a possible relation to the connection of a hypertrophied ligament with prolapsed disc. As a result, we can conclude that the borderline between normal and pathologic LF thickness should not be set at $4 \mathrm{~mm}$. Hypertrophy of the LF above $4 \mathrm{~mm}$ is usually involved in the pathogenesis of lumbar spinal stenosis, which can narrow the diameter of the spinal canal and compress the dural sac and nerve roots, causing many symptoms, even in the absence of a bulging annulus fibrosus or herniated nucleus pulposus or osseous spurs $[1,26]$.

A strong correlation between the patient age and LF thickness at the L4-L5 level has been reported by Okuda et al. [23] and Altinkaya et al. [2], who showed that the LF increase in thickness with age. On the other hand, Safak et al. [26] found no association between LF thickness and the age. Safak et al. [26] suggested that the degradation and mechanical stress are more important than age or gender as risk factors affecting the thickness of the LF.

When the spine is tilted or bent, the LF extends and the tension increases; despite the elastic and flexible nature of the ligament, at neutral position, the ligament has pretension which prevents the possibility to from buckling or wrinkles. Repeated injury can result in initiating many inflammatory and remodelling changes that cause the degeneration, hyperplasia, and hypertrophy of LF. Moreover, the LF may bulge in the canal space and reduce the diameter of the spinal canal, as a result, it would compress the nearby nerves and cause a disturbance in the local circulatory cycle. Some studies had suggested that the degree of hypertrophy of $L F$ is positively correlated with chronic low back pain, and it is difficult to be cured [21]. This means that LF thickness may be closely related to the pathogenesis of spinal pain processes.

All above mentioned delineated the important of identifying the normal LF thickness, since the thickening or hypertrophy of the LF can lead to spinal stenosis and narrowing the spinal canal, it may compress the intraspinal nerve root or cauda equine. This stenosis, compression and corresponding neural symptoms can even occur in the absence of articular process hyperplasia or nucleus pulposus prolapse. Moreover, this study is of paramount importance due to the significant increase in the incidence of lumbar spinal narrowing, as LF hypertrophy is a common cause of lumbar stenosis and is thought to be de- generation-driven pathology, which leads to negative impacts on the quality of life of these patients [22] due to lower limb and lower back pain, numbness, and weakness. These above mentioned symptoms will be mitigated after squatting down or resting.

\section{CONCLUSIONS}

Exploring the correlation between various factors and LF thickness provides reference for pedicle screw placement and lumbar decompression surgery, the development of individualised surgical programmes, and can effectively reduce the incidence of unnecessary postoperative complications induced by misplacement.

\section{Conflict of interest: None declared}

\section{REFERENCES}

1. Abbas J, Hamoud K, Masharawi YM, et al. Ligamentum flavum thickness in normal and stenotic lumbar spines. Spine (Phila Pa 1976). 2010; 35(12): 1225-1230, doi: 10.1097/ BRS.0b013e3181 bfca15, indexed in Pubmed: 20216339.

2. Altinkaya N, Yildirim T, Demir S, et al. Factors associated with the thickness of the ligamentum flavum: is ligamentum flavum thickening due to hypertrophy or buckling? Spine (Phila Pa 1976). 2011; 36(16): E1093-E1097, doi: 10.1097/BRS.0b013e318203e2b5, indexed in Pubmed: 21343862.

3. Berry JA, Elia C, Saini HS, et al. A review of lumbar radiculopathy, diagnosis, and treatment. Cureus. 2019; 11(10): e5934, doi: 10.7759/cureus.5934, indexed in Pubmed: 31788391.

4. Chaves H, Bendersky M, Goñi R, et al. Lumbosacral plexus root thickening: Establishing normal root dimensions using magnetic resonance neurography. Clin Anat. 2018; 31(6): 782-787, doi: 10.1002/ca.23073, indexed in Pubmed: 29575220.

5. Coombs BD, Szumowski J, Coshow W. Two-point Dixon technique for water-fat signal decomposition with B0 inhomogeneity correction. Magn Reson Med. 1997; 38(6): 884-889, doi: $10.1002 / \mathrm{mrm} .1910380606$, indexed in Pubmed: 9402188.

6. Dyck P, Thaisetthawatkul P. Lumbosacral plexopathy. CONTINUUM: Lifelong Learning in Neurology. 2014; 20: 1343-1358, doi: 10.1212/01.con.0000455877.60932.d3.

7. Grenier N, Kressel HY, Schiebler ML, et al. Normal and degenerative posterior spinal structures: MR imaging. Radiology. 1987; 165(2): 517-525, doi: 10.1148/radiology.165.2.3659376, indexed in Pubmed: 3659376.

8. Halil C, Atilla K, Gulis K, et al. Lumbosacral conjoined root anomaly. anatomical considerations of exiting angles and root thickness, results of 21 patients. Turkish Neurosurg. 2015, doi: 10.5137/1019-5149.jtn.16490-15.1.

9. Horwitz T. Lesions of the intervertebral disk and ligamentum flavum of the lumbar vertebrae. Surgery. 1939; 6: 410-425.

10. Huijgen WHF, van Rijswijk CSP, Bloem JL. Is fat suppression in T1 and T2 FSE with mDixon superior to the frequency 
selection-based SPAIR technique in musculoskeletal tumor imaging? Skeletal Radiol. 2019; 48(12): 1905-1914, doi: 10.1007/s00256-019-03227-8, indexed in Pubmed: 31154494.

11. Iwanaga J, Simonds E, Patel M, et al. Anatomic study of superior cluneal nerves: application to low back pain and surgical approaches to lumbar vertebrae. World Neurosurg. 2018; 116: e766-e768, doi: 10.1016/j.wneu.2018.05.087, indexed in Pubmed: 29787877.

12. Izci $Y$, Gürkanlar D, Ozan $H$, et al. The morphological aspectsof lumbar plexus and roots. Turkish Neurosurg. 2005; 15(2): 87-92.

13. Khasawneh RR. Influence of age, sex, height and lumber stenosis on the position of the conus medullaris in adults. Int J Morphol. 2019; 37: 867-871.

14. Kirkaldy-Willis WH. The relationship of structural pathology to the nerve root. Spine (Phila Pa 1976). 1984; 9(1): 49-52, doi: 10.1097/00007632-198401000-00010, indexed in Pubmed: 6719256.

15. Ko HY, Park JH, Shin YB, et al. Gross quantitative measurements of spinal cord segments in human. Spinal Cord. 2004; 42(1): 35-40, doi: 10.1038/sj.sc.3101538, indexed in Pubmed: 14713942.

16. Kolte VS, Khambatta S, Ambiye MV. Thickness of the ligamentum flavum: correlation with age and its asymmetry-an magnetic resonance imaging study. Asian Spine J. 2015; 9(2): 245-253, doi: 10.4184/asj.2015.9.2.245, indexed in Pubmed: 25901237.

17. Kosaka H, Sairyo K, Biyani A, et al. Pathomechanism of loss of elasticity and hypertrophy of lumbar ligamentum flavum in elderly patients with lumbar spinal canal stenosis. Spine (Phila Pa 1976). 2007; 32(25): 2805-2811, doi: 10.1097/ BRS.0b013e31815b650f, indexed in Pubmed: 18246001.

18. Lee S, Choi DS, Shin HS, et al. FSE T2-weighted two-point Dixon technique for fat suppression in the lumbar spine: comparison with SPAIR technique. Diagn Interv Radiol. 2018; 24(3): 175-180, doi: 10.5152/dir.2018.17320, indexed in Pubmed: 29770772.

19. Liu Lm, Song Ym, Gong Q. [Treatment of lumbar stenosis and root pain resulting from simple hypertrophy of lumbar ligamentum flavum]. Zhongguo Xiu Fu Chong Jian Wai Ke Za Zhi. 2003; 17(1): 50-51, indexed in Pubmed: 12916310.

20. Liu Y, Zhou X, Ma J, et al. The diameters and number of nerve fibers in spinal nerve roots. J Spinal Cord Med. 2015; 38(4):
532-537, doi: 10.1179/1079026814Z.000000000273, indexed in Pubmed: 24605949.

21. Munns JJ, Lee JYB, Espinoza Orías AA, et al. Ligamentum flavum hypertrophy in asymptomatic and chronic low back pain subjects. PLoS One. 2015; 10(5): e0128321, doi: 10.1371/journal. pone.0128321, indexed in Pubmed: 26010138.

22. Nandi J, Chowdhery A. A randomized controlled clinical trial to determine the effectiveness of caudal epidural steroid injection in lumbosacral sciatica. J Clin Diagn Res. 2017; 11(2): RC04-RC08, doi: 10.7860/JCDR/2017/21905.9392, indexed in Pubmed: 28384946.

23. Okuda T, Fujimoto $\mathrm{Y}$, Tanaka N, et al. Morphological changes of the ligamentum flavum as a cause of nerve root compression. Eur Spine J. 2005; 14(3): 277-286, doi: 10.1007/s00586-004-0782-5, indexed in Pubmed: 15583951.

24. Ramani PS, Perry RH, Tomlinson BE. Role of ligamentum flavum in the symptomatology of prolapsed lumbar intervertebral discs. J Neurol Neurosurg Psychiatry. 1975; 38(6): 550-557, doi: 10.1136/jnnp.38.6.550, indexed in Pubmed: 1151422.

25. Reeder SB, Pineda AR, Wen Z, et al. Iterative decomposition of water and fat with echo asymmetry and least-squares estimation (IDEAL): application with fast spin-echo imaging. Magn Reson Med. 2005; 54(3): 636-644, doi: 10.1002/mrm.20624, indexed in Pubmed: 16092103.

26. Safak $A A$, Is $M$, Sevinc $O$, et al. The thickness of the ligamentum flavum in relation to age and gender. Clin Anat. 2010; 23(1): 79-83, doi: 10.1002/ca.20883, indexed in Pubmed: 19941359.

27. Sinnatamby C, Last R. Last's anatomy regional and applied. Edinb Churchill Livingstone Elsevier 2011.

28. Yoshida M, Shima K, Taniguchi $Y$, et al. Hypertrophied ligamentum flavum in lumbar spinal canal stenosis. Pathogenesis and morphologic and immunohistochemical observation. Spine (Phila Pa 1976). 1992; 17(11): 1353-1360, doi: 10.1097/00007632-199211000-00015, indexed in Pubmed: 1462211.

29. Yusof MI, Hassan MN, Abdullah MS. The relationship amongst intervertebral disc vertical diameter, lateral foramen diameter and nerve root impingement in lumbar vertebra. Malays Orthop J. 2018; 12(1): 21-25, doi: 10.5704/MOJ.1803.004, indexed in Pubmed: 29725508. 